\title{
The Loss of Information Due to Finite Sample Volume in Radar-Measured Reflectivity
}

\author{
I. I. ZAWADZKI \\ Dept. of Meteorology, McGill University, Monireal, Quebec, Canada
}

(Manuscript received 15 November 1972, in revised form 19 February 1973)

ABSTRACT

\begin{abstract}
The decrease of the mean square and the variance of reflectivity due to radar beam smoothing and post-detection integration are studied in terms of the autocorrelation function of the field of reflectivity. An exponential form of this function is used to evaluate the results. Applications to the problem of radar beam resolution and design of radar displays are discussed.
\end{abstract}

\section{Introduction}

The effect of finite beam width on the slopes of measured reflectivity profiles and the locations of echo tops has been studied by Donaldson (1964). In fact, any information about storm structure will be affected, to a certain degree, by smoothing by the radar beam and in post-detection integration. It is the purpose of this paper to study this problem in a general way, expressing the effect of smoothing by the reduction in the variance of the field of reflectivity.

Fig. 1 illustrates how the reflectivity field is modified by the radar system for a hypothetical profile of reflectivity factor $Z$ (Fig. 1a), averaged over a small volume $^{1}$ (say $10 \times 10 \times 10 \mathrm{~m}$ ), along an arbitrary direction. Since, in general, $Z$ could vary appreciably within the beam width and the pulse length, the radar at best will detect a smoothed version of $Z$, denoted by $Z_{0}$, as shown by the dashed trace in Fig. 1b. At the same time the relative motion of the individual scatterers and their random distribution of phase cause fluctuations in time and in space to appear in the signal. To eliminate these, some kind of combination of independent data is usually made (Marshall and Hitschfeld, 1954; Kodaira, 1959; Smith, 1966; Lhermitte and Kessler, 1966). We will only consider the integration in space and the resulting loss of small-scale variability, as in Fig. 1c, which can be characterized quantitatively by the reduction in the variance of the reflectivity factor. In this example, as well as in the rest of the paper, the effect of smoothing on the signal fluctuations is ignored, and only the reduction of variance due to the smoothing

\footnotetext{
${ }^{1}$ The reflectivity factor is not a continuous function of space since $Z$ results from contributions of individual scatterers. To obtain a continuous field an average over a volume, large enough to contain an appreciable number of scatterers, has to be considered. The particular size given in this example is arbitrary, although adequate for the purpose.
}

of the reflectivity field by the sample volume is considered. In other words, the effect of the smoothing of the reflectivity field by the radar beam and the postdetection integration will be studied independently of the fluctuating nature of the radar signal. This is

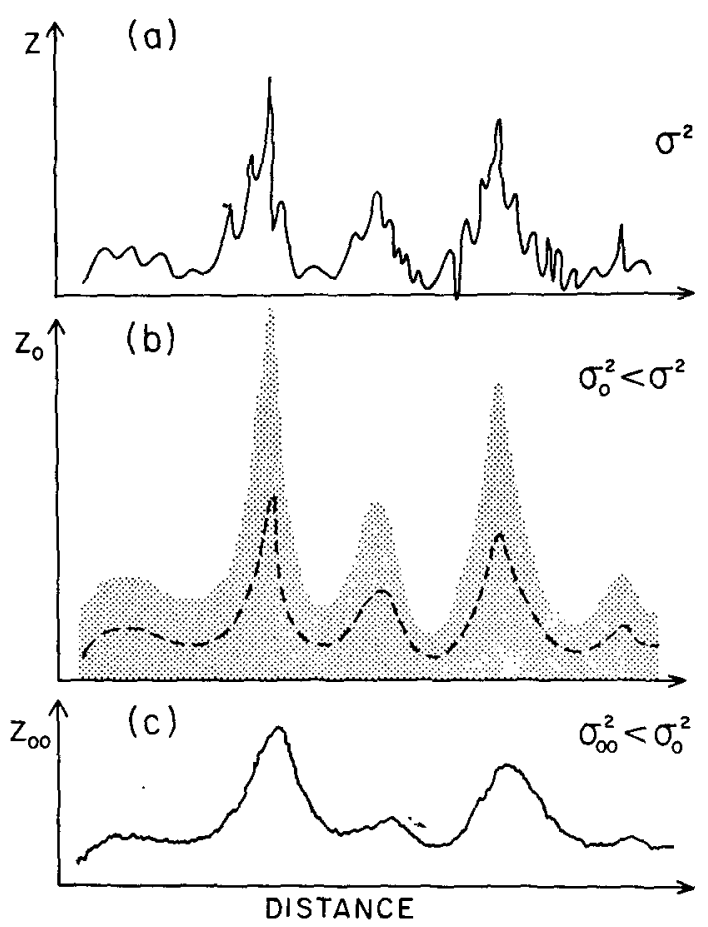

FIG. 1. Effect of the radar system on the reflectivity field for a hypothetical profile of reflectivity factor $Z$ (a). The same profile is shown after detection by the radar receiver in (b) where the dashed curve shows the actual beam-integrated reflectivity factor, although due to the characteristic fluctuations of the radar echo the measured values will have a probability distribution with a standard deviation indicated by the shaded area. In (c) the profile is shown after post-detection integration. 
possible since it is known that after sufficient integration is made, the radar gives a good estimate of the average reflectivity within the sample volume. Accordingly, the problem will be treated as in filtering theory.

\section{Smoothing by the radar beam}

If $\mathbf{r}$ is the vector position in space, let $w(\mathbf{r})$ denote a general normalized smoothing function and $\eta(\mathbf{r})$ a field of reflectivity. When $\eta(\mathbf{r})$ is smoothed by $w(\mathbf{r})$, the resulting smoothed field will be

$$
\eta_{0}(\mathbf{r})=\int_{-\infty}^{\infty}(\mathbf{r}-\mathbf{v}) w(\mathbf{v}) d \mathbf{v} .
$$

It can be shown (Blackman and Tukey, 1959) that if $A(\gamma)$ is the autocorrelation function of $\eta(\mathbf{r})$, the autocorrelation function (ACF) of $\eta_{0}(\mathbf{r})$ is given by

$$
A_{0}(\boldsymbol{\gamma})=\int_{-\infty}^{\infty} A(\boldsymbol{\gamma}-\mathbf{r}) d \mathbf{r} \int_{-\infty}^{\infty} w(\mathbf{v}) w(\mathbf{v}-\mathbf{r}) d \mathbf{v},
$$

where $\gamma$ denotes the vector space lag. It has been verified by Zawadzki (1972) that (2) is applicable to nonstationary, with non-zero mean, processes as long as $A(\gamma)$ is defined over a region containing the entire storm (see Zawadzki, 1973).

Since the value of the ACF for zero lag is the mean square reflectivity, taking $\boldsymbol{\gamma}=0$ in (2), we have

$$
\left\langle\eta_{0}{ }^{2}\right\rangle=\left\langle\eta^{2}\right\rangle \int_{-\infty}^{\infty} a(\mathbf{r}) d \mathbf{r} \int_{-\infty}^{\infty} w(\mathbf{v}) w(\mathbf{v}-\mathbf{r}) d \mathbf{v}
$$

where the angle braces indicate a mean value over the entire storm, and $a(\mathbf{r})$ is the normalized ACF of $\eta(\mathbf{r})$. It should be pointed out here that $a(\mathbf{r})$ in (3) is defined without subtracting the mean reflectivity from $\eta(\mathbf{r})$.

To obtain the mean square reflectivity smoothed by the radar beam, we must determine the form of $w(\mathbf{r})$ for this case. For this we note that in spherical coordinates $(r, \theta, \phi)$ the radar equation for a distributed target of reflectivity $\eta(r, \theta, \phi)$ is

$$
\overline{P_{r}}=\frac{P_{t} \lambda^{2}}{64 \pi^{3}} \int_{V} \frac{G^{2}(\theta, \phi) \eta(r, \theta, \phi)}{r^{4}} d V .
$$

where $G(\theta, \phi)$ is the (one-way) antenna gain function, $\lambda$ the wavelength, $r$ the range, $P_{t}$ and $\overline{P_{r}}$ the transmitted and received powers, and the integration is performed over the scattering volume. For a uniform field of reflectivity $\eta_{0}$

$$
\overline{P_{r}}=\frac{P_{1} \lambda^{2}}{64 \pi^{3}} \eta_{0} \int_{V} \frac{G^{2}(\theta, \phi)}{r^{4}} d V .
$$

In fact, the reflectivity is not constant, and by combining (4) and (5) we obtain the reflectivity (uniform within the scattering volume) which gives the same returned power as the actual reflectivity:

$$
\eta_{0}=\int_{V} \frac{G^{2}(\theta, \phi) \eta(r, \theta, \phi)}{r^{4}} d V / \int_{V} \frac{G^{2}(\theta, \phi)}{r^{4}} d V .
$$

As the beam scans the space, for every position of the center of the beam a value of the reflectivity smoothed by the radar beam is obtained.

Let us now consider the following simplified problem. The scattering volume is assumed to move on a horizontal plane, that is, $\phi=0$. The movement of the scattering volume is considered within a small region, so that the displacement can be expressed in rectangular coordinates such that the tangential component of the displacement lies on the $x$ axis, and the radial component on the $y$ axis. The variations with range of the beam width and the transmitted power density are assumed negligible over the extent of the $y$ displacement. In effect, range in (6) is considered constant.

Also, the reflectivity is assumed to be independent of height, which is nearly true under the cloud base. In rectangular coordinates, and with the above assumptions, (6) can be written as

$$
\eta_{0}=\int_{V} G^{2}(x, z) \eta(x, y) d V / \int_{V} G^{2}(x, y) d V .
$$

The scattering volume in the $y$ direction is defined by the transmitted pulse. Assuming that it has a sharp rectangular shape and a length $h$, it can be expressed as

$$
H(y)= \begin{cases}1, & \text { if } \frac{-h}{4} \leqslant y \leqslant \frac{h}{4} \\ 0, & \text { otherwise }\end{cases}
$$

Now expressing $\eta_{0}$ as a function of the coordinates of the center of the scattering volume $(u, v)$, and with the aid of (8), we can write (7) as a convolution integral:

$$
\eta_{0}(u, v)=\frac{\iint_{-\infty}^{\infty} \int H(y) \mathrm{G}^{2}(x, z) \eta(x-u, y-v) d x d y d z}{\iiint_{-\infty}^{\infty} G^{2}(x, y) H(y) d x d y d z}
$$

The limits of integration in (9) are not inconsistent with the assumption of small displacements since $G^{2}(x, z)$ and $H(y)$ are negligible or zero outside a small region.

By comparing (9) and (1) we see that the smoothing function associated with the two-dimensional smoothing by the radar beam is

$$
w(x, y)=\frac{H(y)}{B} \int_{-\infty}^{\infty} G^{2}(x, z) d z,
$$


with the normalization constant

$$
B=\iint_{-\infty}^{\infty} \int G^{2}(x, z) H(y) d x d y d z
$$

If the antenna gain function is approximated by a Gaussian expression

$$
G(x, y)=G_{0} \exp \left(-\frac{x^{2}}{2 b^{2}}-\frac{z^{2}}{2 c^{2}}\right)
$$

where $b$ and $c$ are related to the (one way) half-power beam widths $\theta_{1}$ and $\phi_{1}$ by

$$
b^{2}=\frac{r^{2} \theta_{1}^{2}}{8 \ln ^{2}}, \quad c^{2}=\frac{r^{2} \phi_{1}^{2}}{8 \ln ^{2}}
$$

and (10) and (11) are used to evaluate (3), the resulting expression is

$\frac{\left\langle\eta_{0}{ }^{2}\right\rangle}{\left\langle\eta^{2}\right\rangle}=\frac{4}{\sqrt{2} \pi h^{2} b} \int_{-\infty}^{\infty} \int_{-h / 2}^{h / 2} a(x, y)\left(\frac{h}{2}-|y|\right) e^{-x^{2} / 2 b^{2}} d x d y$.

Since the smoothing function given by (10) introduces no bias, the variance of the smoothed field is

$$
\sigma_{0}^{2}=\left\langle\eta_{0}^{2}\right\rangle-\langle\eta\rangle^{2}
$$

From (12) and (13) the variance of the reflectivity smoothed by the radar beam can be obtained in terms of the mean, mean square, and the $\mathrm{ACF}$ of reflectivity in the unsmoothed field.

To evaluate (12) the ACF of the unsmoothed reflectivity field is needed. A very dense network of raingages could be used to obtain this ACF if values of rainfall rates are converted to values of reflectivity using a $Z-R$ relationship. Alternatively, time records of a single raingage can be used for this purpose if time is converted to space using the velocity of the storm and assuming the validity of the Taylor hypothesis (see, for example, Lumley and Panofsky, 1964) for short periods of time. Preliminary work of this type with one summers' records of three raingages by Zawadzki (1972) showed that the normalized ACF of reflectivity can be approximated by an exponential of the form

$$
a(x, y)=\exp \left[-\frac{\left(x^{2}+y^{2}\right)^{\frac{1}{2}}}{D}\right]
$$

with the value of $D$ in the order of $0.5 \mathrm{~km}$ for summer storms.

Using (14), Eq. (12) was evaluated; the results are shown in Fig. 2 as a function of the beam width relative to $D$ in the lower scale and of the beam width for $D=0.5$ $\mathrm{km}$ in the upper. For $r \theta_{1}<0.05 \mathrm{~km}$ and $h / D<0.6$, the mean square reflectivity is reduced by beam smoothing

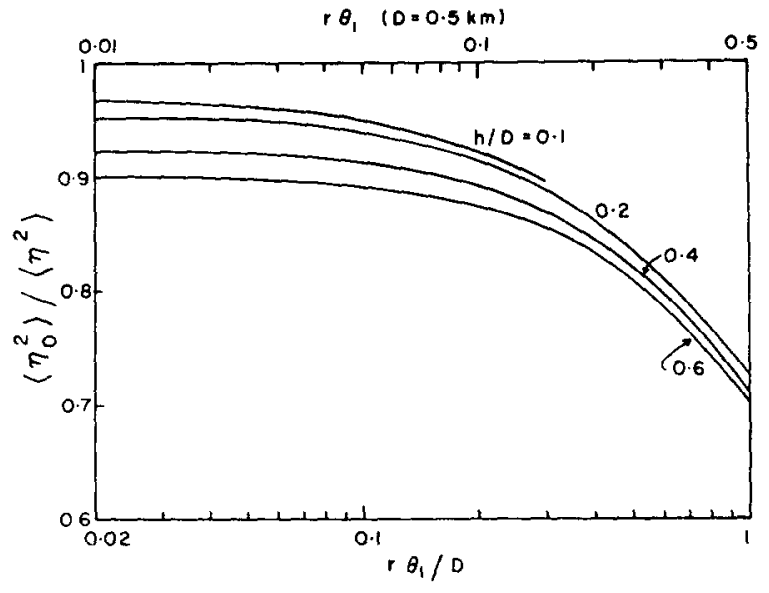

FIG. 2. Reduction in mean square reflectivity due to radar beam smoothing as function of beam width relative to the decorrelation distance of the field of reflectivity.

by less than $10 \%$. For larger values of $r \theta_{1},\left\langle\eta_{0}^{2}\right\rangle /\left\langle\eta^{2}\right\rangle$ decreases rapidly with the beam width. For a $1^{\circ}$ beam at a range of $28 \mathrm{~km}$ the decrease in mean square value is $30 \%$. The pulse length appears to be unimportant whenever the effect of smoothing is appreciable.

The relative decrease in variance is

$$
\frac{\sigma^{2}-\sigma_{0}^{2}}{\sigma^{2}}=\frac{\left\langle\eta^{2}\right\rangle-\left\langle\eta_{0}^{2}\right\rangle}{\left\langle\eta^{2}\right\rangle-\langle\eta\rangle^{2}}
$$

and exceeds the relative decrease in mean square reflectivity

$$
\frac{\left\langle\eta^{2}\right\rangle-\left\langle\eta_{0}^{2}\right\rangle}{\left\langle\eta^{2}\right\rangle}
$$

which is obtainable from the curves in Fig. 2 .

\section{Smoothing in post-detection integration}

Due to the fluctuating nature of radar echoes, data are averaged after detection to reduce the uncertainty in measured reflectivity. Let us consider the case of the square-law detector, in which the quantity obtained after averaging is proportional to the reflectivity. Disregarding the fluctuations of the signal, the radarmeasured reflectivity can be obtained by averaging $\eta_{0}$. If the average is taken over an area $L_{1} \times L_{2}$, the smoothed reflectivity is given by

$$
\eta_{00}=\frac{1}{L_{1} L_{2}} \int_{0}^{L_{1}} \int_{0}^{L_{2}} \eta_{0}(x, y) d x d y .
$$

Let us now define a uniform smoothing function over this area by

$$
w(x, y)=H(x) H(y)
$$




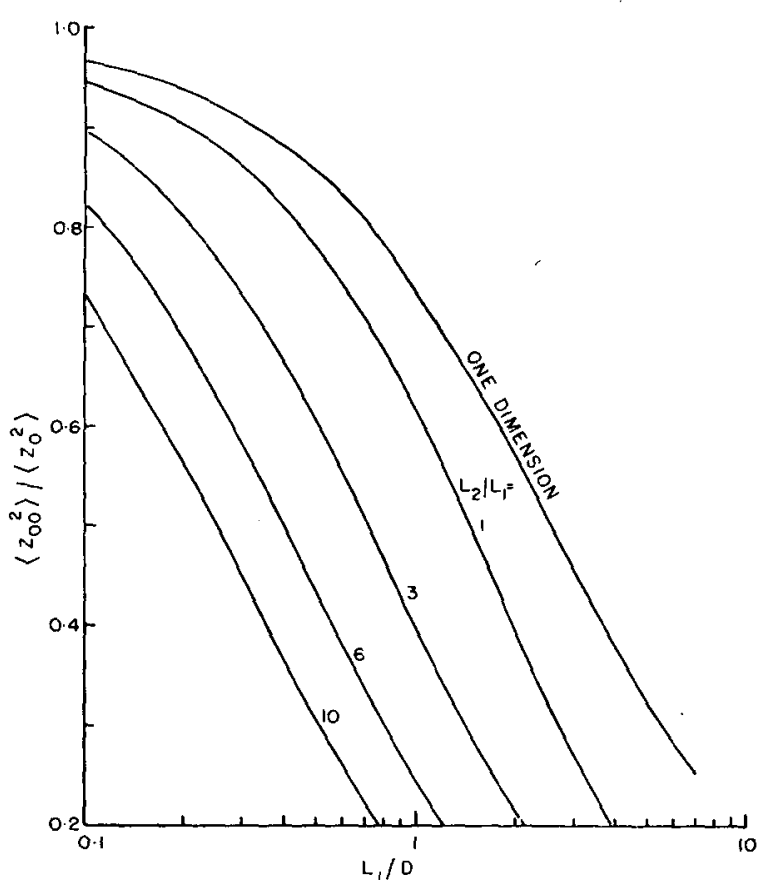

Fig. 3. Rerluction in mean square reflectivity factor due to post-detection integration.

where

$$
\text { v } H(x)= \begin{cases}\frac{1}{L_{1}}, & \text { if }-\frac{L_{1}}{2} \leqslant x \leqslant \frac{L_{1}}{2} \\ 0, & \text { otherwise }\end{cases}
$$

and similarly for $H(y)$. When (3) is evaluated for this smoothing function, the resulting expression is

$$
\begin{aligned}
\frac{\left\langle\eta_{00}{ }^{2}\right\rangle}{\left\langle\eta_{0}{ }^{2}\right\rangle}=\frac{1}{\left(L_{1} L_{2}\right)^{2}} \int_{-L_{1}}^{L_{1}} \int_{-L_{2}}^{L_{2}} a_{0}(x, y) \\
\quad \times\left(L_{1}-|x|\right)\left(L_{2}-|y|\right) d x d y .
\end{aligned}
$$

Here $a_{0}(x, y)$ is the normalized ACF of the field of reflectivity smoothed by the radar beam. Since the reflectivity factor $Z$ is proportional to $\eta$, the normalized ACF's will be identical for both of these quantities and the right-hand side of (16) also gives $\left\langle Z_{00}{ }^{2}\right\rangle /\left\langle Z_{0}^{2}\right\rangle$.

Eq. (16) has been evaluated for the ACF of (14) and the results are plotted in Fig. 3 for various rectangular uniform smoothing functions. Thus, if the field of refectivity is smoothed by averaging over a square of side length equal to the decorrelation distance $\left(L_{1} / D=1\right.$, $L_{2} / L_{1}=1$ ), the mean square value is reduced to 0.61 of its original value (decreased by $39 \%$ ). The reduction in variance in post-detection integration can be obtained from

and Fig. 3.

\section{Discussion}

The computations of the effect of smoothing were made here assuming a symmetrical exponential autocorrelation function. It is important, therefore, to assess the validity of this assumption. The work of Zawadzki (1972, 1973) using radar data indicates that within small scales (of the order of $10 \mathrm{~km}$ ) the precipitation patterns are, in fact isotropic. Although this has been confirmed for only two storms, they were of different types and each one was observed for an extended period of time. Particular regions of precipitation may be strongly anisotropic, but on the average the assumption of a symmetrical form of the autocorrelation function is valid in studies of effects over distances of several kilometers. This consideration makes it possible to extend to two dimensions the values of the autocorrelation function obtained from raingage data in which the time scale is converted to distance in the direction of motion using the velocity of the storm. Work of this type at McGill University using ten years of raingage data shows that the autocorrelation function is either exponential within lags of the order of several kilometers or can be well approximated by a sum of a small number of exponentials of the form

$$
a(x, y)=\sum_{i} c_{i} \exp \left(-\frac{x^{2}+y^{2}}{D_{i}^{2}}\right) .
$$

If (17) is used in (12) or (16), a sum of integrals, with each term containing a simple exponential, is obtained. Figs. 2 and 3 can be thought of as representing each term, and for every value of $D_{i}$ the reduction in the mean square reflectivity (or reflectivity factor) can be obtained from these figures. The combined effect is the weighted sum of the individual results with the weighting factors $c_{i}$.

In light of this brief commentary it is proposed that the results reported here and their possible applications to the interpretation of radar measurements will have a quite general validity.

\section{Some applications of the results}

In attempting to define the resolution of the radar beam one finds that the ability to distinguish two features in the weather depends not only on the beam characteristics and the separation of the features but also on the measurement precision and the prominence of the features with respect to the background weather. The parameter which characterizes this prominence is the variance. Thus, although the question of distinguishing two features is meaningful in a particular case, in general the resolution can be thought of as the ability to detect the variance of the reflectivity field. This idea is particularly useful in the case of widespread weather patterns. The radar beam resolution could be defined quantitatively as the size of a uniform smooth- 
ing area which reduces the variance in the same manner as the radar beam. A comparison between values of $\left\langle Z_{00}{ }^{2}\right\rangle /\left\langle Z_{0}{ }^{2}\right\rangle$ taken from Figs. 2 and 3 show that if $L_{1} / D$ is taken equal to $h / 2 D$ and $L_{2} / D=r \theta / D$, the two values differ very little. For example, for $L_{2} / L_{1}=1$ and $h / D=0.2, r \theta / D$ is equal to 0.1 . For this value, Fig. 2 shows a decrease in mean square value by a factor 0.938 . From Fig. 3, for $L_{2} / L_{1}=1$ and $L_{1} / D=0.1$, the value is 0.942 . For $L_{2} / L_{1}=6$, Fig. 2 yields the value 0.815 for $h / D=0.2$, while from Fig. 4 the value obtained is 0.82 . It was verified that this correspondence between (12) and (16) holds for any value of $h / D$. Since both smoothing functions conserve the mean value it may be concluded that the radar beam reduces the variance as much as a uniform smoothing function of the form of (15a) of width equal to the half-power beam width. Thus, the beam width is a good measure of the resolution, and (16) also gives the reduction in mean square reflectivity due to beam smoothing.

When radar data are combined to reduce the signal fluctuations, a compromise is made between space resolution and precision of the reflectivity measurement. At the same time, the variance of the field is reduced and therefore the detection of extreme values of reflectivity is less likely. When these extreme values are of

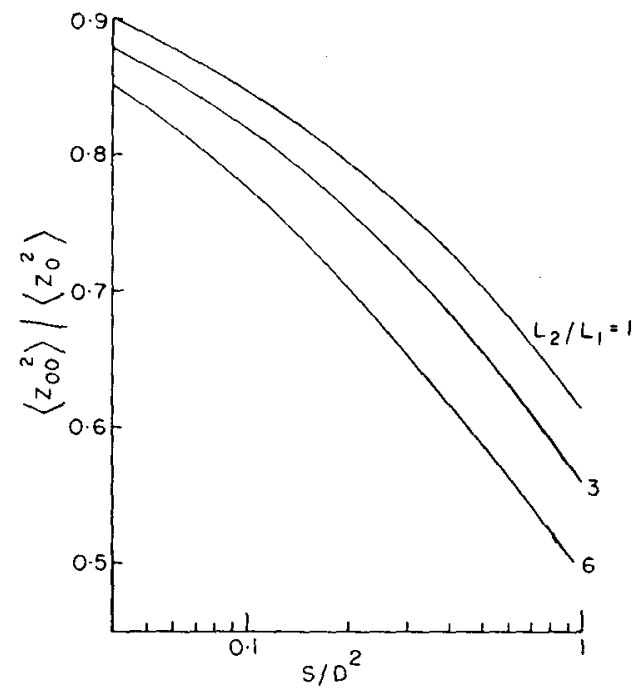

F1G. 4. Effect of the shape of the area over which the post detection is made, on the reduction in the mean square reflectivity. particular interest, as in hail detection, a desired resolution or precision should be set for the three quantities (space, reflectivity, and its variance). It is interesting to note here that integration over areas of square shape reduces the variance slightly less than integration over the same areas of rectangular shape. Values from Fig. 3 have been replotted in Fig. 4 to show the reduction in mean square value as function of area for different shapes. The reduction in variance will be more pronounced. For $S / D^{2}=1$ the mean square reflectivity factor is reduced by $38 \%$ if the integration is performed over a square area $\left(L_{2} / L_{1}=1\right)$, while if the integration is made over the same area but with a rectangular shape such that $L_{2} / L_{1}=6$, the reduction in the mean square value is $50 \%$. Thus, when a radar display is designed, preference should be given to integration over square areas to retain as much as possible of the original variability of the field. Circular areas of integration are probably more effective from this point of view but they are technically impractical.

Acknowledgments. The author is greatly indebted to Dr. R. R. Rogers for many helpful comments. Financial support was provided by a grant from the Atmospheric Environment Service of Canada and a contract from the Canadian Department of Communications.

\section{REFERENCES}

Blackman, R. B., and J. W. Tukey, 1959: The Measurement of Power Spectra. New York, Dover, p. 90.

Donaldson, R. J., Jr., 1964: A demonstration of antenna beam errors in radar reflectivity patterns. J. Appl. Meteor., 3, 611-623.

Kodaira, N., 1959: Quantitative mapping of weather echoes. Res. Rept. No. 30, Dept. of Meteorology, M.I.T.

Lhermitte, R. M., and E. Kessler, 1966: Estimation of the average intensity of precipitation targets. Preprints Twelfth Conf. Radar Meteorology, Norman, Okla., Amer. Meteor. Soc., 23-27.

Lumley, J. L., and H. A. Panofsky, 1964: The Structure of Almospheric Turbulence. New York, Wiley, p. 56.

Marshall, J. S., and W. Hitschfeld, 1954: Interpretation of the fluctuating echo from randomly distributed scatterers: Part I. Can. J. Pliys., 31, 962-1009.

Smith, P. L., 1966: Interpretation of the fluctuating echo from randomly distributed scatterers: Part 3. Preprints Twelfth Conf. Radar Meleorology, Norman, Okla., Amer. Meteor. Soc., $1-6$.

Zawadzki, I. I., 1972: Statistical studies of radar precipitation patterns. Ph.D. thesis, McGill University.

, 1973: Statistical properties of precipitation patterns. J. Appl. Meleor., 12, 459-472. 